\title{
The epoxyeicosatrienoic acid-stimulated phos- phorylation of EGF-R involves the activation of metalloproteinases and the release of HB-EGF in cancer cells
}

\author{
Li-ming CHENG ${ }^{1, \#}$, Jian-gang JIANG ${ }^{1, \#}$, Zi-yong SUN ${ }^{1}$, Chen $\mathrm{CHEN}^{1}$, Ryan T DACKOR ${ }^{2}$, Darryl C ZELDIN², Dao-wen WANG ${ }^{1, *}$ \\ ${ }^{1}$ The Institute of Hypertension and Department of Internal Medicine, Tongji Hospital, Tongji Medical College, Huazhong University of \\ Science and Technology, Wuhan 430030, China; ${ }^{2}$ Division of Intramural Research, National Institute of Environmental Health Sci- \\ ences, NIH, Research Triangle Park, NC 27709, USA
}

\begin{abstract}
Aim: To test the hypothesis that the epoxyeicosatrienoic acid (EET)-induced transactivation of EGF-R depends on the activation of metalloproteinases and the subsequent release of HB-EGF in cancer cells.

Methods: Exogenous 14,15-EET were added to four human-derived cancer cell lines Tca-8113, A549, HepG2, and MDA-MB-231, or these same cell lines were transfected with a mutant CYP epoxygenase (CYP102 F87V, an active 14,15-epoxygenase). The effects of elevated EET levels on the phosphorylation of tyrosine residues in the EGF receptor and on ERK1/2 activation were then assessed. Results: Both the addition of 14,15-EET and the transfection of cells with CYP102 F87V markedly increased the phosphorylation of the tyrosine residues of EGF-R and ERK1/2, an effect that was blocked by a selective EGF-R tyrosine kinase inhibitor (tyrphostin AG1478), a broad-spectrum metalloproteinase inhibitor (1,10-phenanthroline), and an inhibitor of HB-EGF release (CRM197) in Tca-8113 cells. In addition, AG1478, 1,10-phenanthroline, and CRM197 also inhibited the tyrosine phosphorylation of EGF-R and ERK1/2 that was induced by 14,15-EET in the A549, HepG2, and MDA-MB-231 cell lines.

Conclusion: These results suggest that the EET-induced transactivation of EGF-R depends on activation of metalloproteinases and the subsequent release of HB-EGF in cancer cell lines.
\end{abstract}

Keywords: arachidonic acid; cytochrome P450 epoxygenase; epoxyeicosatrienoic acids; tumor cell proliferation; EGF-R; ERK1/2; AG1478; phenanthroline; CRM197; metalloproteinase

Acta Pharmacologica Sinica (2010) 31: 211-218; doi: 10.1038/aps.2009.184

\section{Introduction}

Arachidonic acid is a polyunsaturated fatty acid that is a component of membrane phospholipids and is acutely released in response to exposure to a number of agonists, including growth factors, cytokines, and hormones, in various cell types. Upon release, it is metabolized via the cyclooxygenase, lipoxygenase, or cytochrome P450 (CYP) monooxygenase pathways, which yield prostaglandins, leukotrienes, and epoxyeicosatrienoic acids (EETs), respectively ${ }^{[1,2]}$. When arachidonic acid is metabolized by CYP epoxygenases, it results in the generation of four regioisomeric EETs $(5,6-, 8,9-, 11,12-$, and 14,15-

\footnotetext{
\# The first two authors contributed equally to this work.

* To whom correspondence should be addressed.

E-mail dwwang@tjh.tjmu.edu.cn

Received 2009-07-18 Accepted 2009-11-19
}

EET ${ }^{[3,4]}$. We and others have demonstrated that CYP-derived EETs induce changes that have protective effects in endothelial cells, including the upregulation of endothelial nitric oxide synthase (eNOS) expression and activity, the enhancement of angiogenesis, an increase in fibrinolytic activity via the production of tissue plasminogen activator (tPA), and the inhibition of TNF-a-induced apoptosis. Additionally, CYP-derived EETs possess anti-inflammatory properties ${ }^{[3,5]}$. Recently, EETs have been implicated in a variety of physiologic processes that are relevant to cancer pathogenesis, including the regulation of intracellular signaling pathways, gene expression, tumor cellular proliferation, and metastasis via the activation of the MAPK and PI3 kinase/Akt pathways as well as via the phosphorylation of EGF-R ${ }^{[6,7]}$.

In renal proximal tubular epithelial cells, it was found that stable CYP102 F87V expression induced 14,15-EET produc- 
tion and enhanced cellular proliferation ${ }^{[3]}$. Further studies in these cells demonstrated that 14,15-EET induced the activation of matrix metalloproteinases (MMPs), which in turn led to the release of heparin-binding epidermal growth factor-like growth factor (HB-EGF), a potent mitogenic EGF-R ligand ${ }^{[3]}$. In human cancer cells, however, it remains unknown whether the EGF-R phosphorylation and the malignant proliferation induced by CYP epoxygenase-derived EETs involve the activation of MMPs and/or HB-EGF release. A better understanding of the mitogenic signaling events that are initiated by CYP epoxygenase-EET signaling may lead to the development of new anti-cancer therapeutic strategies. Therefore, we investigated the effects that exogenous 14,15-EET and CYP epoxygenase overexpression had on MMP activation and EGF-R phosphorylation in the human cancer cell lines.

\section{Materials and Methods Materials}

Tca-8113 (a human tongue squamous carcinoma cell line), A549 (a human lung cancer cell line), HepG2 (a human liver cancer cell line), MDA-MB-231 (a human breast cancer cell line) were obtained from the American Type Culture Collection (Manassas, VA). Chemicals and reagents were obtained as follows: Cell culture medium were purchased from Hyclone (Logan, UT); antibodies against epidermal growth factor receptor (EGF-R) and phospho-EGF-R from Cell Signaling Technology (Beverly, MA); anti-extracellular signalregulated kinase (ERK) and anti-phospho-ERK antibodies from New England Biolabs, Inc (Beverly, MA); antibodies against $\beta$-actin from Neomarkers (Fremont, CA); horseradish peroxidase-conjugated secondary antibodies (goat anti-rabbit immunoglobulin $\mathrm{G}$ and rabbit anti-mouse immunoglobulin $\mathrm{G}$ ) from KPL (Gaithersburg, MD); enhanced chemiluminescence reagents from Pierce, Inc (Rockford, IL); 14,15-EET from Cayman Chemical Co (Ann Arbor, MI); AG1478 (EGF-R-selective tyrosine kinase inhibitor) from Calbiochem (San Diego, CA); and EGF, 1,10-phenanthroline (a non-specific MMP inhibitor) and CRM197 (an inhibitor of HB-EGF release) from Sigma Chemical Co (St Louis, MO). All other reagents were purchased from standard commercial suppliers.

\section{Adeno-associated virus (AAV)-mediated CYP epoxygenase over- expression}

The recombinant adeno-associated virus ( $\mathrm{HAAV}$ ) vector pXXUF1, packaging plasmid pXX2, adenovirus helper plasmid pXX6, and an rAAV plasmid containing the GFP cDNA (GFP-pUF1) were generous gifts from Dr Xiao XIAO (University of Pittsburgh, Pittsburgh, PA). CYP102 F87V is a mutant $\mathrm{P} 450$ that is derived from Bacillus megaterium ( $\mathrm{P}_{50} 0_{\mathrm{BM} 3}$ ) in which phenylalanine 87 is replaced with valine, converting it to a highly regio- and stereoselective epoxygenase that biosynthesizes 14(S),15(R)-EET from arachidonic acid. The coding region of the CYP102 F87V mutant was subcloned into pXXUF1 downstream from the cytomegalovirus promoter to produce the construct CYP102 F87V-pUF1. The rAAVs were produced and purified as previously described ${ }^{[3,8,9]}$ and their titers were determined by dot blot hybridization. The eluted rAAV was then aliquoted and stored at $-80{ }^{\circ} \mathrm{C}$. The resultant rAAVs were designated rAAV-CYP102 F87V and rAAV-GFP. Next, Tca-8113 cells were infected with rAAV-CYP102 F87V or rAAV-GFP (about 50 virions/cell) and cultured for one week to allow them to obtain specifying expression. These cells were used for further experiments as detailed below.

\section{Cell culture and cell proliferation assays}

Tca-8113 cells were cultured at $37^{\circ} \mathrm{C}$ in DMEM containing $10 \%(v / v)$ bovine serum, $100 \mathrm{mg} / \mathrm{mL}$ streptomycin, and 100 $\mathrm{IU} / \mathrm{mL}$ penicillin in a humidified atmosphere containing $5 \%$ $\mathrm{CO}_{2}$. The cells were seeded onto 96-well plates (about $1 \times 10^{4} /$ well) in DMEM containing 10\% bovine serum in a final volume of $0.2 \mathrm{~mL}$. Six parallel wells were set up for control and treated group. Once the cells reached $60 \%$ confluence, the medium was changed to serum-free DMEM and the cells were incubated at $37^{\circ} \mathrm{C}$ for $12 \mathrm{~h}$ to allow for synchronization. Next, 14,15-EET ( $250 \mathrm{nmol} / \mathrm{L})$ and AG1478 (100 nmol/L) were added to the medium with ethanol used as the vehicle control. After 24 h, 3-(4,5-dimethylthiazol-2-yl)-2,5-diphenyltetrazolium bromide (MTT) $(20 \mu \mathrm{L} /$ well of $5 \mathrm{mg} / \mathrm{mL}$ in PBS) was added to the culture medium and the cells were incubated at $37^{\circ} \mathrm{C}$ for 4 more hours, at which point the reaction was stopped by the addition of $100 \mu \mathrm{L}$ DMSO. The reaction product was then quantified by measuring the absorbance at 490 $\mathrm{nm}$ using an ELISA reader (Elx800, China).

\section{Immunoblot analysis}

Tca-8113, A549, HepG2, and MDA-MB-231 cells were treated with 14,15-EET $(250 \mathrm{nmol} / \mathrm{L})$ or EGF $(20 \mathrm{ng} / \mathrm{mL})$ with and without various inhibitors, including AG1478 $(100 \mathrm{nmol} / \mathrm{L})$, CRM197 $(10 \mu \mathrm{g} / \mathrm{mL})$, and 1,10-phenanthroline $(100 \mu \mathrm{mol} / \mathrm{L})$. The rAAV-infected cells were treated with the inhibitors AG1478 (100 nmol/L), CRM197 (10 $\mu \mathrm{g} / \mathrm{mL})$, and 1,10-phenanthroline $(100 \mu \mathrm{mol} / \mathrm{L})$. Cells were then harvested for Western blot analysis to allow the detection of signaling molecules. Cells were placed on ice, washed twice with ice-cold PBS, and then lysed in RIPA lysis buffer containing $50 \mathrm{mmol} / \mathrm{L}$ Tris ( $\mathrm{pH}$ 8.0 ), $150 \mathrm{mmol} / \mathrm{L} \mathrm{NaCl}, 0.1 \%$ sodium dodecyl sulfate (SDS), $1 \%$ Nonidet P-40 (NP-40), $0.5 \%$ sodium deoxycholate, $0.02 \%$ sodium azide $\left(\mathrm{NaN}_{3}\right), 1 \mu \mathrm{g} / \mathrm{mL}$ aprotonin, and $1 \mathrm{mmol} / \mathrm{L}$ phenylmethylsulfonyl fluoride. Solubilized lysates were centrifuged at $10000 \times \mathrm{g}$ for $15 \mathrm{~min}$. Total protein concentrations were determined by the Bradford assay. After the supernatants were heated to $95^{\circ} \mathrm{C}$ for $10 \mathrm{~min}$, they were electrophoresed on SDS-PAGE $(8 \%-12 \%)$ gradient gels and transferred to PVDF membranes. Blots were incubated overnight at $4{ }^{\circ} \mathrm{C}$ with primary antibodies and washed 4 times with TBST before they were probed with horseradish peroxidase-conjugated secondary antibodies for $2 \mathrm{~h}$ at room temperature. Blots were then visualized with enhanced chemiluminescence reagent and the optical densities of the bands were semi-quantified by the Gene Genius Bio Imaging System (SynGene, USA). In some cases, blots were stripped and reprobed with other antibodies. 
Statistical analysis

Data were expressed as the mean \pm SEM for at least three separate experiments. Statistical evaluation was performed using Student's t-test or ANOVA, as was appropriate for the situation. $P$-values less than 0.05 were considered to be statistically significant.

\section{Results}

14,15-EET induces the phosphorylation of EGF-R and ERK1/2

When cells were incubated with 14,15-EET, there was a dosedependent increase in the phosphorylation of EGF-R and ERK1/2 observed (Figure 1A and 1B), a finding that had been previously demonstrated by our group ${ }^{[6]}$. We treated cells with the EGF-R-selective tyrosine kinase inhibitor tyrphostin AG1478 to examine its effect on the EET-induced phosphorylation of EGF-R and ERK1/2. Our results showed that AG1478 attenuated the EET-induced phosphorylation of EGF-R and ERK1/2 (Figure 1C). As expected, AG1478 also attenuated the EGF-induced phosphorylation of EGF-R and ERK1/2 (Figure 1D). These results demonstrate that 14,15-EET enhances the phosphorylation and activation of ERK1/2 in Tca-8113 cells by inducing the transactivation of EGF-R.

14,15-EET promotes cancer cell proliferation via EGF-R activation We next examined whether 14,15-EET-stimulated proliferation of Tca-8113 cells occurred via the transactivation of EGF-R. The MTT assays showed that 14,15-EET (250 nmol/L) significantly increased Tca-8113 cell proliferation as compared to the vehicle control ( $26 \%$ increase, $P<0.05)$. AG1478 completely abolished the proliferative effect induced by 14,15-EET $(P<0.05$, Figure 2$)$. These results suggest that 14,15 -EET promotes the proliferation of Tca-8113 cells through transactivation of EGF-R.

\section{Effects of 1,10-phenanthroline and diphtheria toxin/CRM197 on} 14,15-EET-induced EGF-R activation

To evaluate whether the activation of EGF-R in Tca-8113 cells resulted from the release of HB-EGF due to proteolytic processing by MMPs, we investigated the effects that 1,10-phenanthroline (a non-specific MMP inhibitor) and diphtheria toxin/ CRM197 (an inhibitor of HB-EGF release) had on EGF-R and ERK1/2 phosphorylation. The MMP inhibitor 1,10-phenanthroline is a heterocyclic organic compound that forms strong complexes with most metal ions and thus acts as a non-specific metalloproteinases inhibitor ${ }^{[10,11]}$. The transmembrane precursor of HB-EGF (pro-HB-EGF) also serves as the unique highaffinity receptor for diphtheria toxin. CRM197, a nontoxic and catalytically inactive (Glu-52) mutant of diphtheria toxin that binds to the extracellular domain of HB-EGF and spe-

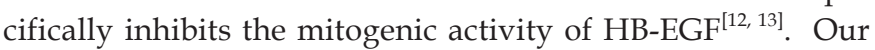
results showed that when Tca- 8113 cells were treated with 1,10-phenanthroline (100 $\mathrm{mmol} / \mathrm{L}), 14,15$-EET-induced EGF-R and ERK1/2 phosphorylation were completely blocked, but that EGF-induced EGF-R and ERK1/2 phosphorylation was not inhibited (Figure 3A and 3B). Furthermore, pretreatment of Tca-8113 cells with CRM197 $(10 \mu \mathrm{g} / \mathrm{mL})$ led to a significant
A

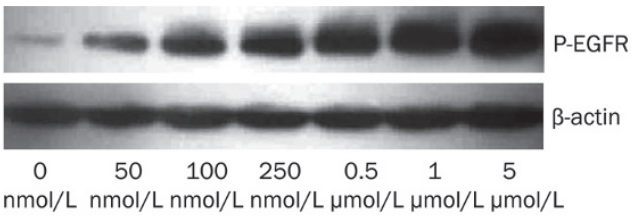

B

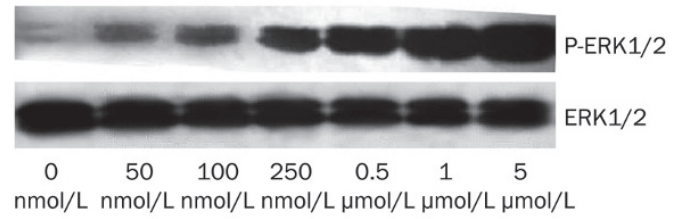

C
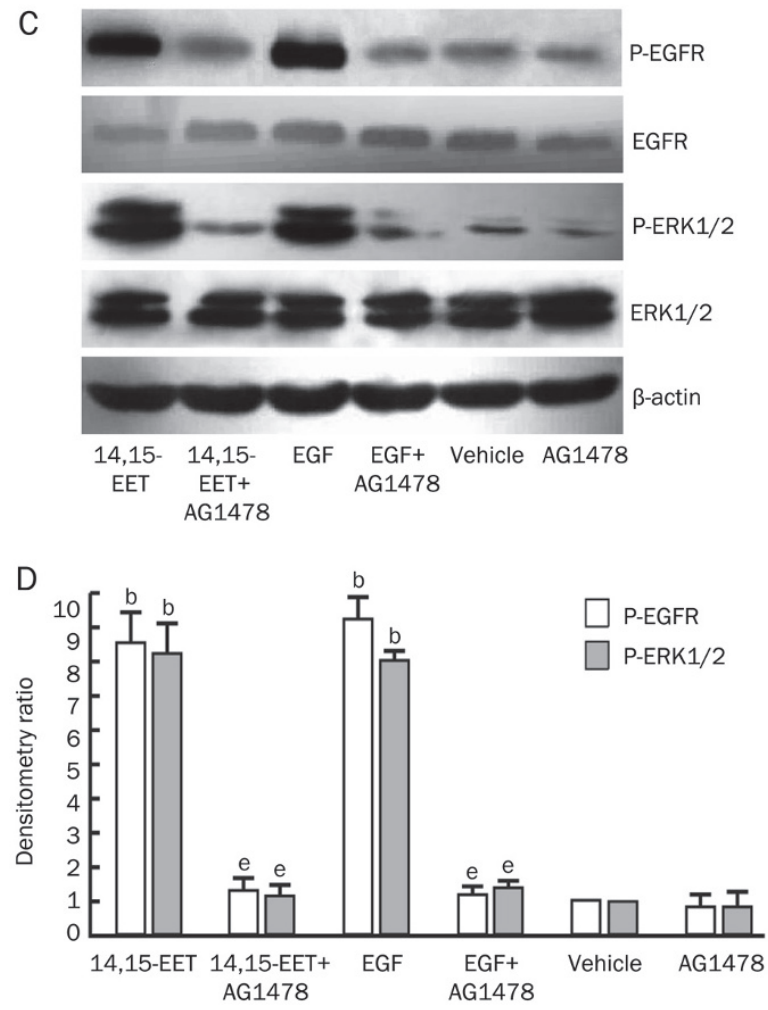

Figure 1. 14,15-EET increases the tyrosine phosphorylation of EGF-R and ERK1/2 in a dose-dependent manner. Serum-deprived Tca-8113 cells were treated with vehicle (ethanol) or different concentrations of 14,15 -EET $(50,100,250,500 \mathrm{nmol} / \mathrm{L}, 1$, and $5 \mu \mathrm{mol} / \mathrm{L})$ for 30 min. (A) Phosphorylation of EGF-R was detected using a site-specific tyrosine antibody to EGF-R; $\beta$-actin was used as a loading control. (B) Phosphorylated ERK1/2 and total ERK1/2 were analyzed by Western blots. (C) and (D) Effect of tyrphostin AG1478 on EGF-R and ERK1/2 phosphorylation. Serum-deprived Tca-8113 cells were pretreated with or

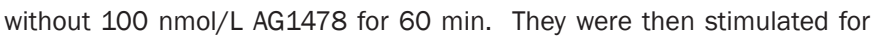
$30 \mathrm{~min}$ with $250 \mathrm{nmol} / \mathrm{L}$ 14,15-EET or $20 \mathrm{ng} / \mathrm{mL}$ EGF. Phosphorylated EGF-R, total EGF-R, phosphorylated ERK1/2, and total ERK1/2 were detected using appropriate antibodies. (C) Representative Western blots of three separate experiments with similar results. (D) Quantification of three separate experiments by densitometry. ${ }^{\mathrm{b}} P<0.05$ vs vehicle control group; ${ }^{\mathrm{e}} P<0.05$ vs no signaling pathway inhibitor group.

inhibition of the phosphorylation of EGF-R and ERK1/2 by 14,15-EET. However, CRM197 pretreatment had no effect on the EGF-induced phosphorylation of these molecules (Figure 


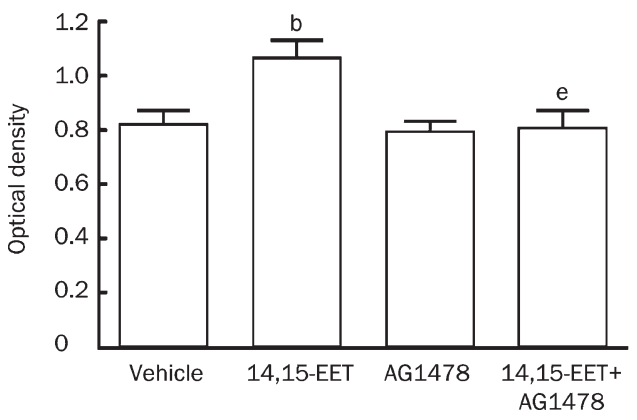

Figure 2. The proliferative effect of 14,15 -EET in Tca- 8113 cells is abrogated by AG1478. Treatment with 14,15-EET ( $250 \mathrm{nmol} / \mathrm{L})$ stimulated Tca-8113 cell proliferation. Cell proliferation was $26 \%$ higher in the treatment group than in the vehicle control group $\left({ }^{b} P<0.05\right)$. Pretreatment with AG1478 completely abolished the proliferative effect induced by 14,15-EET ( $\left.{ }^{\mathrm{P}} \mathrm{P}<0.05\right)$.

3C and 3D). These results are consistent with those obtained with tyrphostin AG1478 and show that the activation of MMPs and subsequent release of HB-EGF are important steps in the phosphorylation of EGF-R and ERK1/2 that is induced by
14,15-EET.

To further investigate the possible mechanism by which 14,15-EET transactivates EGFR, we examined the effects of AG1478, 1,10-phenanthroline, and CRM197 on 14,15-EETinduced EGF-R activation in three other tumor cell lines: A549, HepG2, and MDA-MB-231. As expected, we observed a similar profile to the one we noted in the experiments described above: 14,15-EET increased the levels of phosphorylated EGFR and ERK1/2 in these tumor cells and the 14,15-EET inducedphosphorylation of EGF-R and ERK1/2 was dramatically attenuated by AG1478, 1,10-phenanthroline, and CRM197 (Figure 4). There was no synergistic effect observed when 1,10-phenanthroline and CRM197 were added in combination. These data further indicate that the release of HB-EGF and activation of MMPs both exert regulatory effects on the phosphorylation of EGF-R and ERK1/2 that is induced by 14,15EET in tumor cells.

\section{Effects of 14,15-epoxygenase CYP102 F87V on the transactiva- tion of EGF-R}

We and others have demonstrated that CYP102 F87V is a 14,15-epoxygenase that efficiently metabolizes arachidonic

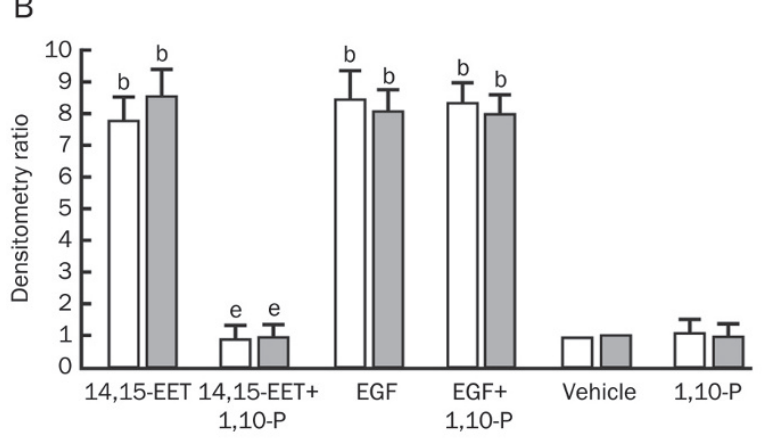

\section{C}

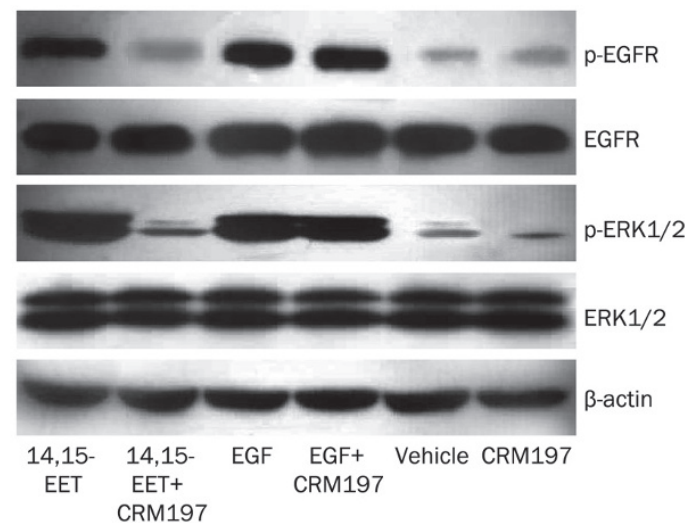

D

A
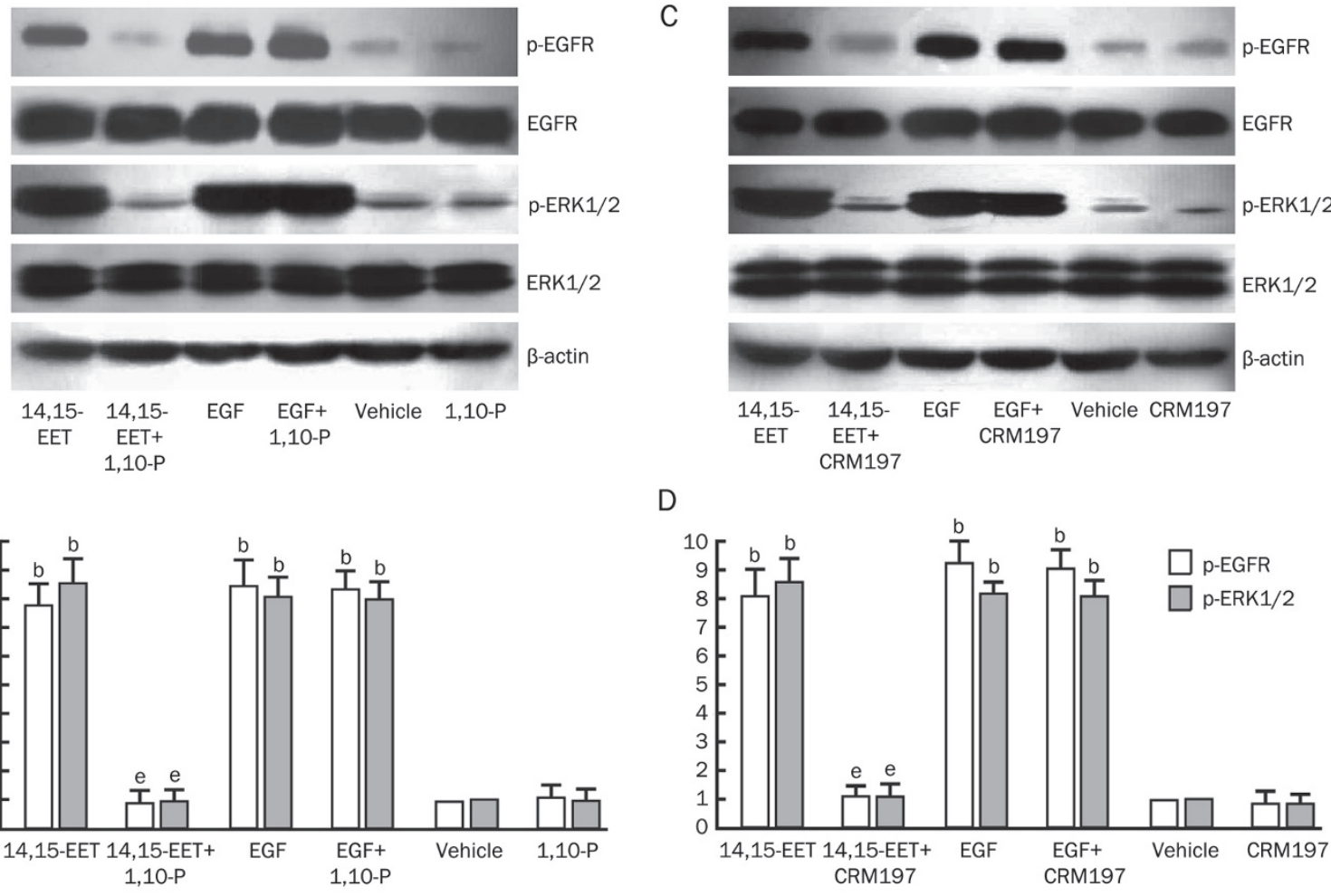

Figure 3. Effects of 1,10-phenanthroline and CRM197 on the 14,15-EET-induced tyrosine phosphorylation of EGF-R and ERK1/2 in Tca-8113 cells. Serum-deprived Tca-8113 cells were incubated with or without $100 \mu \mathrm{mol} / \mathrm{L}$ of 1,10-phenanthroline or $10 \mu \mathrm{g} / \mathrm{mL}$ of CRM197 for $60 \mathrm{~min}$. They were then stimulated for $30 \mathrm{~min}$ with $250 \mathrm{nmol} / \mathrm{L}$ 14,15-EET or $20 \mathrm{ng} / \mathrm{mL}$ EGF. Phosphorylated EGF-R, total EGF-R, phosphorylated ERK1/2, and total ERK1/2 were detected with the corresponding antibodies. (A) Results of incubation with 1,10-phenanthroline. The results shown are representative of three separate experiments with similar results. (B) Densitometric quantification of three separate experiments examining the effects of incubation with 1,10-phenanthroline. (C) Results of incubation with CRM197. The results shown are representative of three separate experiments with similar results. (D) Densitometric quantification of three separate experiments examining the effects of incubation with CRM197. ${ }^{\mathrm{b}} P<0.05$ vs vehicle control group; ${ }^{e} P<0.05$ vs no signaling pathway inhibitor group. 
A
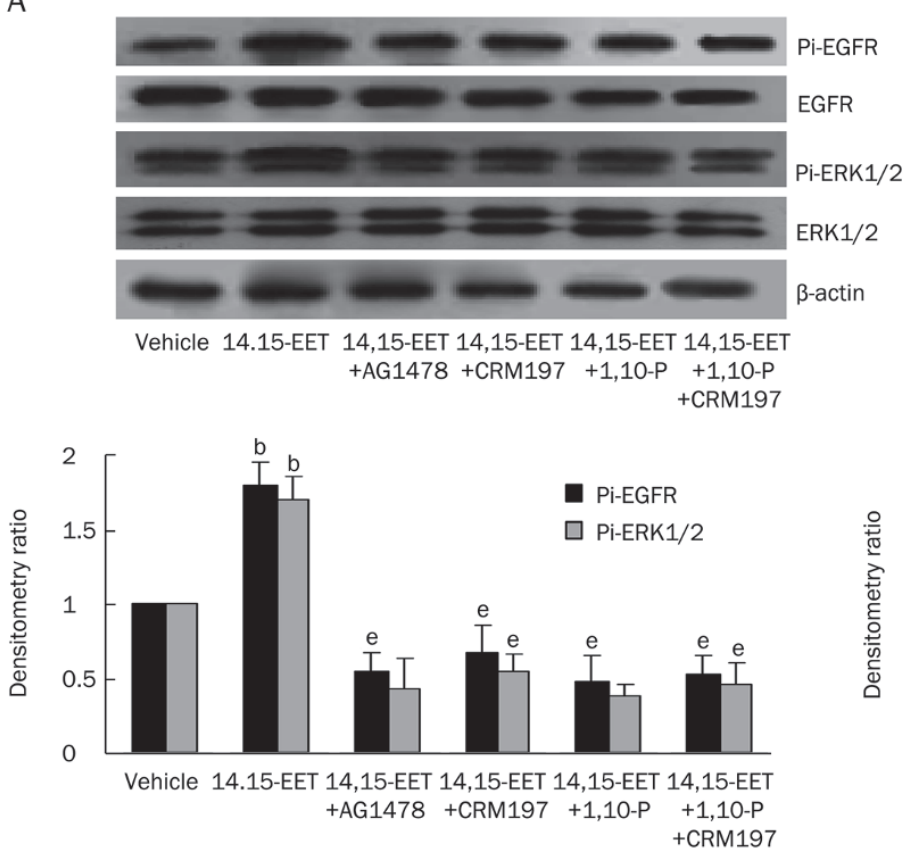

C
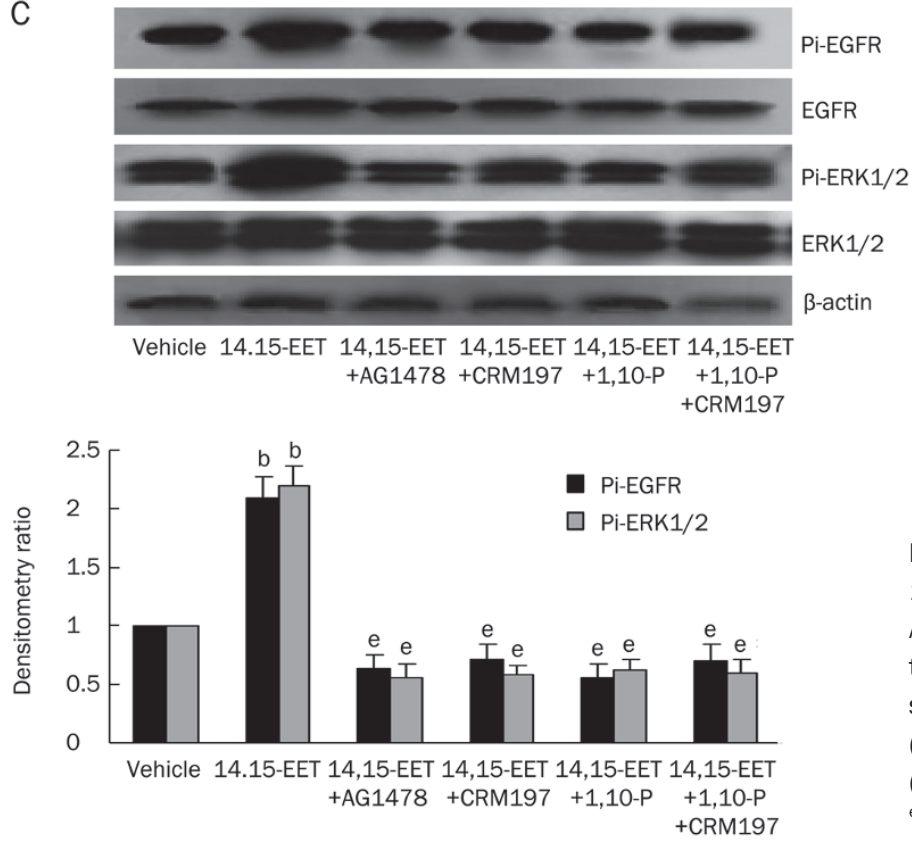

acid to $14,15-\mathrm{EET}^{[6]}$. In this study, we infected Tca-8113 cells with rAAV-CYP102 F87V to determine if CYP epoxygenase overexpression could transactivate EGF-R by activation of the HB-EGF-shedding mechanism. The efficiency of gene transfer was evaluated one week after infection. To assess EET levels, we determined the concentration of its stable metabolites. Western blot analysis revealed robust CYP102 F87V expression in Tca-8113 cells infected with rAAV-CYP102 F87V (Figure 5A). Moreover, we examined the activity of CYP102 F87V in rAAV-CYP102 F87V infected cells. Given the instability of 14,15-EET, the levels of 14,15-DHET(the stable metabolites of
B
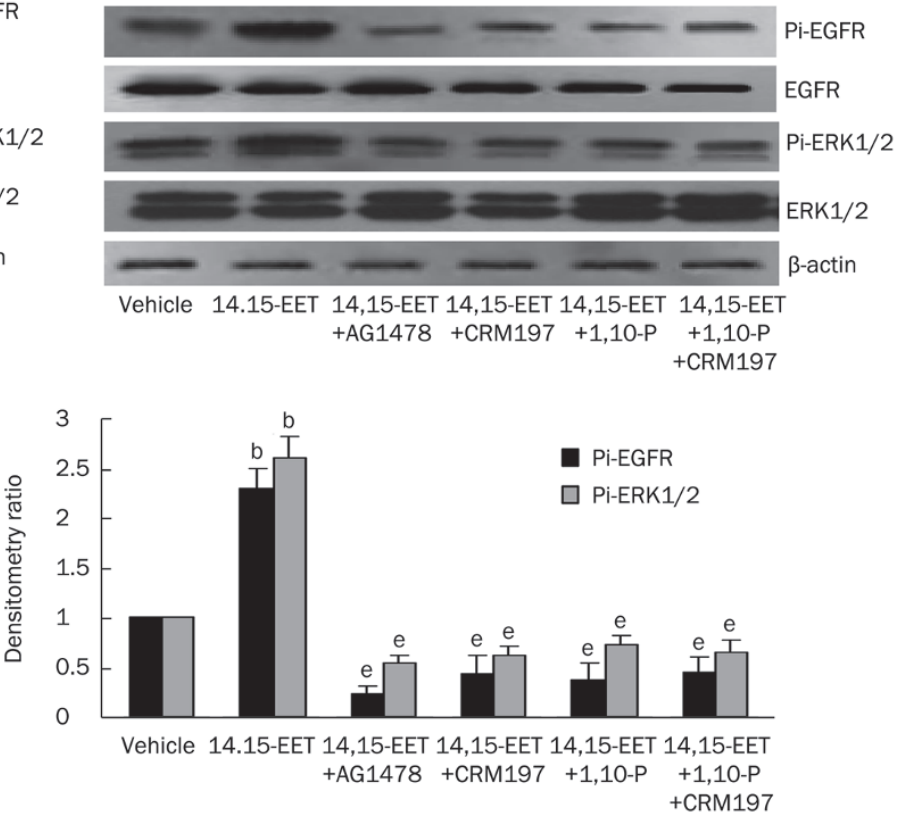

Figure 4. Effects of AG1478, CRM197, and 1,10-phenanthroline on the 14,15-EET-induced tyrosine phosphorylation of EGF-R and ERK1/2 in the A549, HepG2, and MDA-MB-231 cell lines. The upper panel is representative of three separate experiments with similar results. The lower panel shows the quantification of three separate experiments by densitometry. (A) Results from the A549 cell line. (B) Results from the HepG2 cell line. (C) Results from the MDA-MB-231 cell line. ${ }^{\mathrm{b}} \mathrm{P}<0.05$ vs vehicle group; ${ }^{\mathrm{e}} P<0.05$ vs $14,15-\mathrm{EET}$ group.

14,15-EET) were assessed using an ELISA assay. The results showed that there was a dramatic increase in 14,15-DHET levels in rAAV-CYP102 F87V infected cells as compared to untreated cells $(332 \pm 117 \mathrm{pg} / 250 \mu \mathrm{g}$ protein vs $122 \pm 4 \mathrm{pg} / 250$ $\mu \mathrm{g}$ protein, $P<0.05$ ) (Figure 5B). rAAV-CYP102 F87V infection significantly promoted phosphorylation of EGF-R and ERK1/2, a finding that we had observed previously ${ }^{[6]}$. Importantly, the induction of phosphorylation conferred by CYP102 F87V overexpression was abolished by the addition of AG1478 $2 \mathrm{~h}$ before cell harvesting (Figure 6). Furthermore, EGF-R and ERK1/2 phosphorylation were also blocked when CYP102 
A

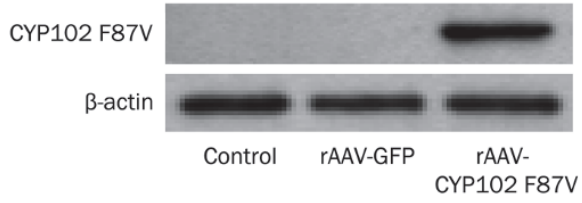

B

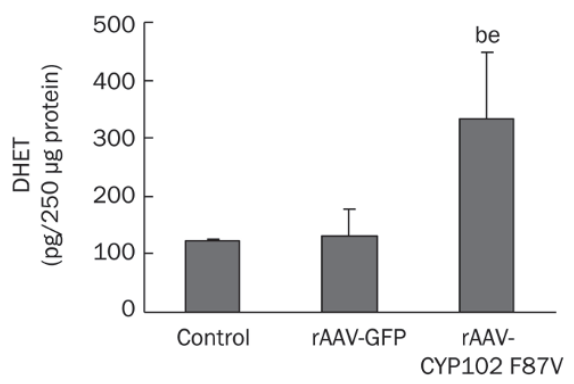

Figure 5. Expression of CYP102 in tumor cells after rAAV-CYP102 F87V gene transfer. (A) CYP102 F87V expression in rAAV-CYP102 F87V-infected cells. (B) CYP102 F87V activity (14,15-DHET production) in rAAV-CYP102 F87V-infected cells. Data are presented as mean \pm SEM. ${ }^{\mathrm{b}} P<0.05$ vs control; ${ }^{\mathrm{e}} P<0.05$ vs rAAV-GFP.

F87V-infected Tca-8113 cells were treated with 1,10-phenanthroline or CRM197 (Figure 6).

\section{Discussion}

Multiple studies have shown EETs to be potent mitogens that activate EGF-R and ERKs in various cell types ${ }^{[3,6]}$. Previous studies by our group have also demonstrated that EETs increased the expression levels and activity of MMPs in various human cancer cell lines ${ }^{[7]}$. However, the role of EETs in the activation of EGF-R in human cancer cells remains unclear. In the present study, we found that the addition of 14,15-EET or the overexpression of a selective 14,15-EET epoxygenase can induce the activation of EGF-R and ERK1/2 in multiple human-derived cancer cell lines: Tca-8113, A549, HepG2, and MDA-MB-23. These signaling events were abolished by the addition of the tyrosine kinase inhibitor of EGF-R, AG1478. Interestingly, the addition of 1,10-phenanthroline (a non-specific MMP inhibitor) or diphtheria toxin/CRM197 (an inhibitor of HB-EGF release) also blocked the EET-induced activation of EGF-R and ERK1/2. As expected, neither the inhibition of MMP activity nor the inhibition of HB-EGF cleavage blocked the EGF-induced EGF-R phosphorylation or its downstream activation of ERK1/2 in the cancer cell lines tested. Together, these results demonstrate that MMP activation with subsequent HB-EGF cleavage and release is essential for EETinduced EGF-R activation in human cancer cells.

HB-EGF is synthesized as a type I transmembrane protein in a manner that is similar to the way in which other members of the epidermal growth factor (EGF) family are synthesized. Pro-HB-EGF can be enzymatically shed within the juxtamembrane region, leading to the release of a soluble 14-22 $\mathrm{kDa}$ growth factor ${ }^{[13]}$. The ectodomain shedding of proHB-EGF is induced by various stimuli, including phorbol ester 12-O-tetradecanoylphorbol-13-acetate (TPA) ${ }^{[14,15]}$ and calcium ionophore ${ }^{[16]}$ as well as various growth factors and cytokines $^{[17]}$. G-protein coupled receptor (GPCR) agonists also stimulate pro-HB-EGF shedding, which in turn mediates EGF-R transactivation via GPCR signaling ${ }^{[18,19]}$. Metalloproteinases have been shown to be responsible for the proteolytic cleavage of pro-HB-EGF because the ectodomain shedding of pro-HB-EGF is efficiently inhibited by various metalloproteinase inhibitors. Protein kinase $\mathrm{C}(\mathrm{PKC})$ and mitogen-activated protein kinase (MAPK) may be required for the activation of appropriate metalloproteinases as they are known to be involved in the intracellular signaling pathway that leads to pro-HB-EGF shedding ${ }^{[20,21]}$.

In human colon carcinoma cells, IL-8 promotes cell proliferation and migration via the metalloproteinase-induced cleavage of pro-HB-EGF ${ }^{[22]}$. Additionally, it has been shown that lysophosphatidic acid (LPA)-induced ectodomain shedding of pro-HB-EGF is critical for tumor formation in ovarian cancer $^{[23]}$, and that the deoxycholic taurine (DCT)-induced transactivation of EGF-R is mediated by the MMP-7-catalyzed release of the EGF-R ligand HB-EGF in H508 human colon cancer cells $^{[24]}$. In this study, we demonstrated that the addition of 14,15-EET as well as the overexpression of a 14,15-EETspecific epoxygenase leads to EGF-R transactivation via MMP activation and HB-EGF release in four cancer cell lines. However, the specific metalloproteinase responsible for the 14,15EET-induced cleavage of pro-HB-EGF has not yet been identified and thus requires further study.

The transactivation of EGF-R and the subsequent activation of downstream ERKs by the metalloproteinase-mediated release of soluble HB-EGF plays an important role in EETstimulated mitogenic signaling. The tyrosine kinase inhibitor of EGF-R, AG1478, may have significant therapeutic value in controlling the malignant growth of various cancers. However, as demonstrated in previous studies, EETs activate the PI3K/Akt signaling pathway in different cell lines and tissues, which can also lead to mitogenic effects ${ }^{[3,6]}$. The relative importance that the PI3K/Akt and EGF-R/ERK/MMP signaling pathways have in mediating the mitogenic effects of the products of CYP epoxygenase remains to be determined.

In summary, this study reveals that EGF-R transactivation is a crucial event in the mitogenic signaling pathways initiated by EETs in cancer cells. In addition, the EET-induced transactivation of EGF-R is mediated by the activation of metalloproteinases that cleave pro-HB-EGF from the cell membrane and release active HB-EGF, which subsequently binds to EGF-R and activates downstream ERKs. Thus, CYP epoxygenasederived EETs may lead to the malignant proliferation of cancer cells as a result of EGF-R transactivation via an MMP-HBEGF signaling pathway. Further studies will be required to identify the precise metalloproteinases that are activated by EETs in cancer cells and to elucidate the relative importance that EGF-R/ERK/MMP and other signaling pathways have in mediating the mitogenic effects of the products of CYP epoxygenase. 
A
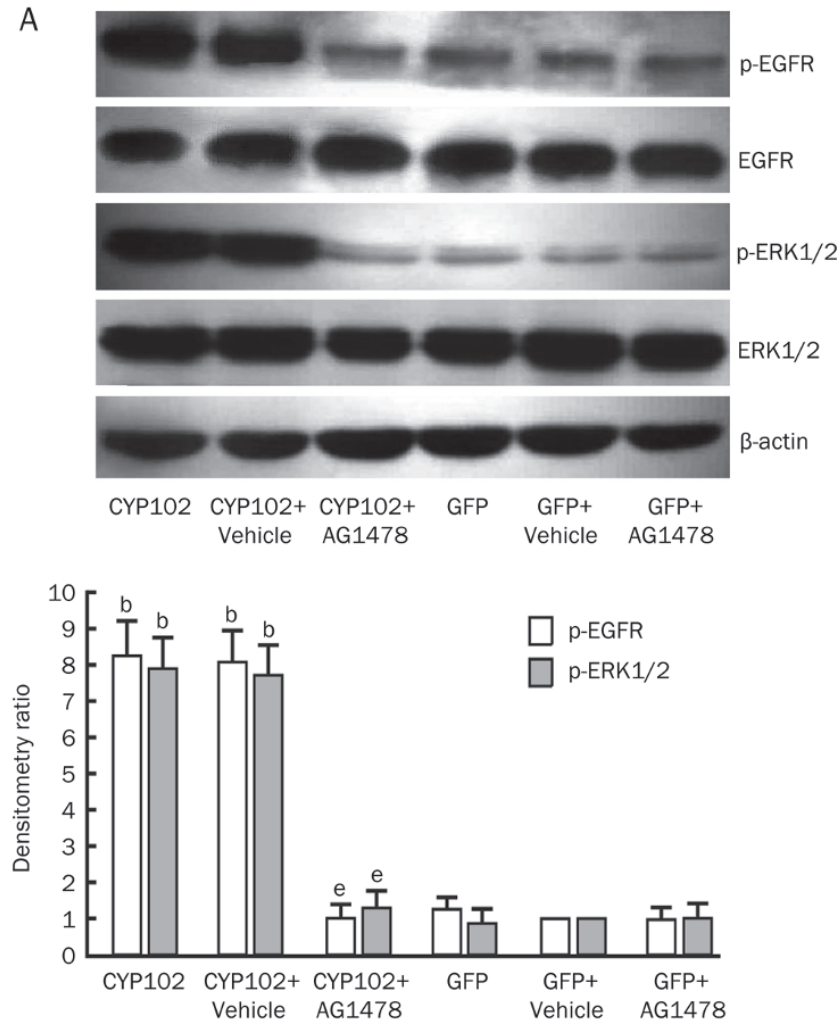

C
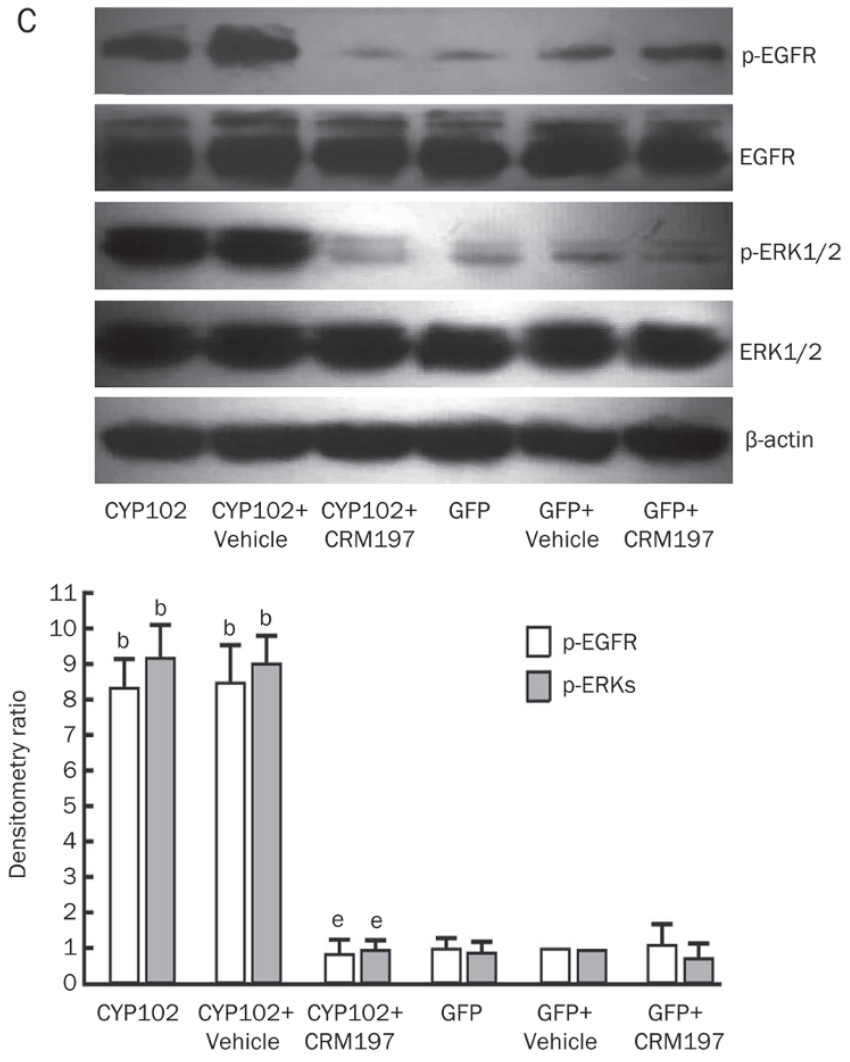

Acknowledgements

This work was supported by the International Collaboration Project (No 2005DFA30880), the 973 grant (2007CB512004),

B
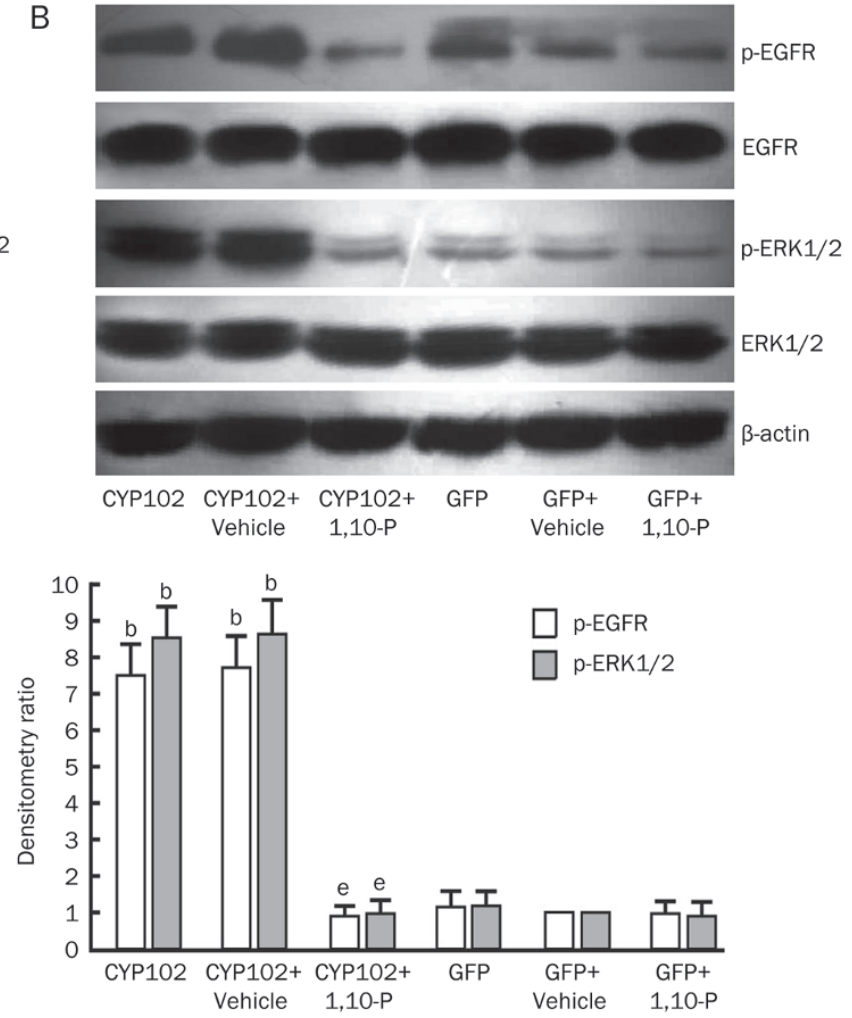

Figure 6. Effects of tyrphostin AG1478, 1,10-phenanthroline, and the nontoxic mutant of diphtheria toxin CRM197 on the activation of EGF-R and ERK1/2 in rAAV-CYP102 F87V-transfected Tca-8113 cells. Tca8113 cells were transfected with rAAV-CYP102 F87V or rAAV-GFP (GFP) and cultured for one week to allow for maximal expression. Serumstarved cells were treated with the indicated agents and cell lysates were immunoblotted with the indicated antibodies. Upper panels are representative of three separate experiments with similar results. Lower panels show the quantification of three separate experiments by densitometry. (A) Results shown are for tyrphostin AG1478. (B) Results shown are for 1,10-phenanthroline. (C) Results shown are for CRM197. ${ }^{\mathrm{b}} \mathrm{P}<0.05$ vs rAAV-GFP control group; ${ }^{\mathrm{e}} \mathrm{P}<0.05$ vs no signaling pathway inhibitor group.

and two National Natural Science Foundation of China grant (30430320, 30770882). This work was also funded, in part, by the Intramural Research Program of the NIH and the National 
Institute of Environmental Health Sciences (Z01 ES025034).

\section{Author contribution}

Li-ming CHENG, Dao-wen WANG designed research; Liming CHENG, Jian-gang JIANG, Chen CHEN performed research; Zi-yong SUN, Ryan T DACKOR, Darryl C ZELDIN contributed new analytical tools and reagents; Li-ming CHENG, Dao-wen WANG, Ryan T DACKOR, Darryl C ZELDIN analyzed data; Li-ming CHENG, Dao-wen WANG wrote the paper.

\section{Abbreviations}

EET, epoxyeicosatrienoic acid; HETE, hydroperoxyeicosatetraenoic acid; CYP, cytochrome P450; EGF-R, epidermal growth factor receptor; p-EGFR, phosphorylated epidermal growth factor receptor; HB-EGF, heparin-binding epidermal growth factor-like growth factor; TNF-a , tumor necrosis factor- $\alpha$; eNOS, endothelial nitric oxide synthase; tPA, tissue plasminogen activator; MMP, matrix metalloproteinase; ERK, extracellular signal-regulated kinase; p-ERK, phosphorylated extracellular signal-regulated kinase; MAPK, mitogen-activated protein kinase; PI3K, phosphatidylinositol 3-kinase; MTT, 3-(4,5-dimethylthiazol-2-yl)-2,5-diphenyltetrazolium bromide; DMEM, Dulbecco's modified Eagle's medium; DMSO, dimethyl sulfoxide; PBS, phosphatebuffered saline; AG1478, a selective epidermal growth factor receptor blocker; CRM197, an inhibitor of HB- epidermal growth factor release; rAAV, recombinant adeno-associated virus; EGF, epidermal growth factor; SDS, sodium dodecyl sulfate; NP-40, Nonidet P-40; $\mathrm{NaN}_{3}$, sodium azide; TPA, 12-O-tetradecanoylphorbol-13-acetate; GPCR, G-protein coupled receptor; PKC, protein kinase C; LPA, lysophosphatidic acid; DHET, dihydroxyeicosatrienoic acid; DCT, deoxycholic taurine; Akt, cellular homolog of the v-alt oncogene, an S/T protein kinase; $\mathrm{PKB}$, protein kinase $\mathrm{B}$.

\section{References}

1 Funk CD. Prostaglandins and leukotrienes: advances in eicosanoid biology. Science 2001; 294: 1871-5.

2 Kroetz DL, Zeldin DC. Cytochrome P450 pathways of arachidonic acid metabolism. Curr Opin Lipidol 2002; 13: 273-83.

3 Chen C, Li G, Liao W, Wu J, Liu L, Ma D, et al. Selective inhibitors of CYP2J2 related to terfenadine exhibit strong activity against human cancers in vitro and in vivo. J Pharmacol Exp Ther 2009; 329: 90818.

4 Capdevila JH, Falck JR, Harris RC. Cytochrome P450 and arachidonic acid bioactivation. Molecular and functional properties of the arachidonate monooxygenase. J Lipid Res 2000; 41: 163-81.

5 Node K, Ruan XL, Dai J, Yang SX, Graham L, Zeldin DC, et al. Activation of Galpha s mediates induction of tissue-type plasminogen activator gene transcription by epoxyeicosatrienoic acids. J Biol Chem 2001; 276: 15983-9.

6 Jiang JG, Chen CL, Card JW, Yang S, Chen JX, Fu XN, et al. Cytochrome P450 $2 J 2$ promotes the neoplastic phenotype of carcinoma cells and is up-regulated in human tumors. Cancer Res 2005; 65: 4707-15.

7 Jiang JG, Ning YG, Chen C, Ma D, Liu ZJ, Yang S, et al. Cytochrome p450 epoxygenase promotes human cancer metastasis. Cancer Res 2007; 67: 6665-74.

8 Xiao X, Li J, Samulski RJ. Efficient long-term gene transfer into muscle tissue of immunocompetent mice by adeno-associated virus vector. J Virol 1996; 70: 8098-108.
9 Xiao X, Li J, Samulski RJ. Production of high-titer recombinant adenoassociated virus vectors in the absence of helper adenovirus. J Virol 1998; 72: 2224-32.

10 Kiss Z. The zinc chelator 1,10-phenanthroline enhances the stimulatory effects of protein kinase $\mathrm{C}$ activators and staurosporine, but not sphingosine and $\mathrm{H}_{2} \mathrm{O}_{2}$, on phospholipase $\mathrm{D}$ activity in NIH 3 T3 fibroblasts. Biochem J 1994; 298 (Pt 1): 93-8.

11 Chen J, Chen JK, Neilson EG, Harris RC. Role of EGF receptor activation in angiotensin II-induced renal epithelial cell hypertrophy. J Am Soc Nephrol 2006, 17: 1615-23.

12 Naglich JG, Metherall JE, Russell DW, Eidels L. Expression cloning of a diphtheria toxin receptor: identity with a heparin-binding EGF-like growth factor precursor. Cell 1992; 69: 1051-61.

13 Mitamura T, Higashiyama S, Taniguchi N, Klagsbrun M, Mekada E. Diphtheria toxin binds to the epidermal growth factor (EGF)-like domain of human heparin-binding EGF-like growth factor/diphtheria toxin receptor and inhibits specifically its mitogenic activity. J Biol Chem 1995; 270: 1015-9.

14 Goishi K, Higashiyama S, Klagsbrun M, Nakano N, Umata T, Ishikawa $\mathrm{M}$, et al. Phorbol ester induces the rapid processing of cell surface heparin-binding EGF-like growth factor: conversion from juxtacrine to paracrine growth factor activity. Mol Biol Cell 1995; 6: 967-80.

15 Raab G, Higashiyama S, Hetelekidis S, Abraham JA, Damm D, Ono $\mathrm{M}$, et al. Biosynthesis and processing by phorbol ester of the cells surface-associated precursor form of heparin-binding EGF-like growth factor. Biochem Biophys Res Commun 1994; 204: 592-7.

16 Dethlefsen SM, Raab G, Moses MA, Adam RM, Klagsbrun M, Freeman MR. Extracellular calcium influx stimulates metalloproteinase cleavage and secretion of heparin-binding EGF-like growth factor independently of protein kinase C. J Cell Biochem 1998; 69: 143-53.

17 Raab G, Klagsbrun M. Heparin-binding EGF-like growth factor. Biochim Biophys Acta 1997; 1333: F179-99.

18 Daub H, Weiss FU, Wallasch C, Ullrich A. Role of transactivation of the EGF receptor in signalling by G-protein-coupled receptors. Nature 1996; 379: 557-60.

19 Prenzel N, Zwick E, Daub H, Leserer M, Abraham R, Wallasch C, et al. EGF receptor transactivation by G-protein-coupled receptors requires metalloproteinase cleavage of proHB-EGF. Nature 1999; 402: 884-8.

20 Izumi Y, Hirata M, Hasuwa H, Iwamoto R, Umata T, Miyado K, et al. A metalloprotease-disintegrin, MDC9/meltrin-gamma/ADAM9 and PKCdelta are involved in TPA-induced ectodomain shedding of membrane-anchored heparin-binding EGF-like growth factor. EMBO J 1998; 17: 7260-72.

21 Gechtman Z, Alonso JL, Raab G, Ingber DE, Klagsbrun M. The shedding of membrane-anchored heparin-binding epidermal-like growth factor is regulated by the Raf/mitogen-activated protein kinase cascade and by cell adhesion and spreading. J Biol Chem 1999; 274: 28828-35.

22 Itoh Y, Joh T, Tanida S, Sasaki M, Kataok H, Itoh K, et al. IL-8 promotes cell proliferation and migration through metalloproteinase-cleavage proHB-EGF in human colon carcinoma cells. Cytokine 2005; 29 : 275-82.

23 Miyamoto S, Hirata M, Yamazaki A, Kageyama T, Hasuwa H, Mizushima $\mathrm{H}$, et al. Heparin-binding EGF-like growth factor is a promising target for ovarian cancer therapy. Cancer Res 2004; 64: 5720-7.

24 Cheng K, Xie G, Raufman JP. Matrix metalloproteinase-7-catalyzed release of HB-EGF mediates deoxycholyltaurine-induced proliferation of a human colon cancer cell line. Biochem Pharmacol 2007; 73: 1001-12. 